\title{
ÍLEO BILIAR: ENTEROLITOTOMIA VIDEOASSISTIDA
}

\author{
GALLSTONE ILEUS: VIDEOASSISTED ENTEROLITHOTOMY
}

\author{
Álvaro Queiroz de Godoy, TCBC-PR ${ }^{1}$ \\ Oscar Tacla Junior ${ }^{2}$ \\ André Reichert da Silva Godoy ${ }^{3}$
}

\section{INTRODUÇÃO}

O íleo biliar, descrito pela primeira vez por Bartholin em 1654, representa de 1 a $4 \%$ de todos os casos de obstrução intestinal mecânica. Ocorre em conseqüência de uma fístula interna entre algum ponto do sistema biliar (geralmente vesícula) com o tubo digestivo (geralmente o duodeno) ${ }^{1}$.

O íleo biliar se desenvolve em 0,3 a $0,5 \%$ dos portadores de cálculos. Como habitualmente acontece em pacientes idosos a mortalidade é elevada (até $17 \%$ ) ${ }^{1}$ que pode ser atenuada através de um diagnóstico precoce e conduta adequada. Este artigo tem o objetivo de apresentar uma opção terapêutica pouco agressiva, adotada pelos autores em um paciente.

\section{RELATO DO CASO}

Paciente do sexo masculino, 81 anos, já com diagnóstico prévio de colecistopatia litiásica, atendido em caráter de urgência com cólicas, distensão e vômitos.

O padrão radiológico abdominal era de obstrução e a ultra-sonografia demonstrou vesícula biliar pequena sem cálculos estando a via biliar principal normal. Foi submetido à videolaparoscopia que evidenciou bloqueio no hipocôndrio direito, distensão de alças e obstrução de jejuno por cálculo de aproximadamente $3,5 \times 2,5 \mathrm{~cm}$. $\mathrm{O}$ método empregado permitiu a orientação para minilaparotomia no flanco esquerdo, exteriorização da alça e enterotomia para retirada do cálculo. A evolução pós-operatória foi boa tendo ocorrido a alta hospitalar no quinto dia.

\section{DISCUSSÃO}

O mecanismo de formação das fistulas internas é decorrente de processos inflamatórios, geralmente da vesícula, provocados por cálculos impactados no infundídu$10^{2}$. A obstrução mais freqüente é no nível do delgado (jejuno ou íleo) e, raramente, do colon ${ }^{3}$. É rara a obstrução do bulbo duodenal (síndrome de Bouveret) ${ }^{4}$. O tamanho dos cálculos varia de 1,9 a $3,5 \mathrm{~cm}$. O diagnóstico pré-operatório é feito em apenas 50 a $60 \%$ dos casos ${ }^{1}$. Clinicamente há sinais de obstrução intestinal ${ }^{5}$. Radiologicamente a clássica tríade de Riegler (obstrução do intestino delgado, cálculos biliares ectópicos e ar na árvore biliar) auxilia muito no diagnóstico. A ultra-sonografia e a tomografia computadorizada também podem contribuir evidenciando ou não cálculos na vesícula biliar, distensão de alças com líquido no interior, ar nas vias biliares, presença de cálculo biliar à distância e dilatação ou cálculos na via biliar principal. Quando a obstrução é no nível do duodeno ou do cólon os exames endoscópicos poderão ser úteis para diagnóstico e tratamento permitindo a retirada dos cálculos. Exames radiológicos contrastados poderão ser utilizados com ressalvas ${ }^{3}$. Em relação ao tratamento há controvérsias sobre a melhor conduta. $\mathrm{O}$ tratamento pode ser feito de três maneiras ${ }^{2}$ : enterolitotomia exclusiva ou deslocamento do cálculo para o cólon com ou sem fragmentação, cirurgia em duas etapas realizando-se inicialmente a enterolitotomia seguida de colecistectomia e fechamento da fístula três a quatro semanas depois e, finalmente, tratamento completo em etapa única. Considerando que a enterolitotomia isolada pode permitir conseqüências negativas a médio e longo prazos, como repetição do quadro obstrutivo, colecistite, colangite, carcinoma da vesícula biliar ou má absorção associada à fístula biliodigestiva, somente deve ser realizada em pacientes com risco operatório elevado. A cirurgia completa em etapa única tem mortalidade mais elevada. A cirurgia em duas etapas implica os transtornos de duas operações. A enterolitotomia isolada videoassistida permitiu, neste caso, a resolução do quadro, o que, eventualmente, pode representar mais uma vantagem da videolaparoscopia, especialmente nos doentes de alto risco cirúrgico. No entanto deve-se ressaltar que trata-se de um procedimento que não é isento de riscos e deve estar reservado a casos

1. Cirurgião do Aparelho Digestivo do Hospital Evangélico, Santa Casa de Misericórdia e Gastrocentro de Londrina - PR

2. Gastroenterologista do Hospital Evangélico, Santa Casa de Misericórdia e Gastrocentro de Londrina - PR

3. Acadêmico da Faculdade de Medicina da Fundação Educacional Serra dos Órgãos - Teresópolis - RJ

Recebido em 7/12/1199

Aceito para publicação em 29/5/2000

Trabalho realizado no Gastrocentro de Londrina-PR 
rigorosamente selecionados. Há que se considerar também que em situações semelhantes onde a distensão de alças for muito acentuada é mais seguro realizar o pneumoperitônio por via aberta. Também é possível que em alguns casos a identificação do cálculo possa oferecer maior dificuldade exigindo exploração de segmentos mais longos do intestino delgado. Outro aspecto importante é que o procedimento poderá ser realizado exclusivamente por videolaparoscopia com sutura da alça e apenas abertura adicional da parede para retirada do cálculo. Sem a videolaparoscopia tanto o diagnóstico de certeza como o tratamento certamente exigiriam uma laparotomia mais extensa com seus possíveis inconvenientes.

\begin{abstract}
Treatment, morbidity and mortality of patients with gallstone ileus depend on an accurate diagnosis made in time, and also on a more adequate therapeutic option.A detailed clinical evaluation is fundamental for such diagnosis. Complementary exams like a simple radiological study of the abdomen, high and low endoscopies, an ultrasonography, and a tomography can also be performed. The therapeutic options include the removal of the obstructive factor separately, the performance of a treatment in two separate stages, or the performance of a complete treatment (removal of the calculus, cholecystectomy, and the closing of the fistula).This study aims to present a case report of an elderly man of high surgical risk, presenting gallstone ileus. He was submitted to an isolated videoassisted enterolithotomy through a minilaparotomy. Taking into consideration the patient's advanced age and the lack of evidence as to other biliary associated pathologies, the chosen treatment seemed to be a good alternative. The evolution was good, and after an 8 month follow-up the patient was found well and with no biliary symptoms whatsoever.
\end{abstract}

Key Words: Gallstone Ileus; Biliary enteric fistula; Diagnosis; Therapy.

\section{REFERENNCIAS}

1. Rodriguez-Sanjuan JC, Casado F, Fernandez MJ et al. Cholecystectomy and fistula closure versus enterolithotomy alone in gallstone ileus. Br J Surg $1997 ; 84$ (5) : 634 - 637.

2. Schumacher G, Keck H, Neuhaus P. - Die cholecystoduodenale Fistel mit nachfolgendem Gallensteinileus: Fallbeschreibung eines ungewöhnlichen Verlaufs. Zentralbl Chir Leipzig 1996; 121 (5) : 408-411.

3. Pérez-Morera A, Pérez-Díaz D, Calvo Serrano M, et al. Obstrucción aguda de colon secundaria a litiasis biliar. Rev Esp Enf Digest 1996; 88 (11): 805-808.

4. Kosorok P - Bouveret's Syndrome ( Gallstone ileus ) - a minefield. Br J Clin Pract 1996; 50 (1): 59-60.
5. Oikarinen H, Päivänsalo $\mathrm{M}$, Tikkakoski T, Saarela A - Radiological findings in biliary fistula and gallstone ileus. Acta Radiologica 1996; 37 (6) : 917-922.

Endereço para Correspondência:

Dr. Álvaro Queiroz de Godoy

Av. Bandeirantes, 324

86010-010 - Londrina - PR 